\title{
NEW SERIES INVOLVING HARMONIC NUMBERS AND SQUARED CENTRAL BINOMIAL COEFFICIENTS
}

\author{
JOHN MAXWELL CAMPBELL
}

ABSTRACT. Recently, there have been a variety of intriguing discoveries regarding the symbolic computation of series containing central binomial coefficients and harmonictype numbers. In this article, we present a vast generalization of the recently-discovered harmonic summation formula

$$
\sum_{n=1}^{\infty}\left(\begin{array}{c}
2 n \\
n
\end{array}\right)^{2} \frac{H_{n}}{32^{n}}=\frac{\Gamma^{2}\left(\frac{1}{4}\right)}{4 \sqrt{\pi}}\left(1-\frac{4 \ln (2)}{\pi}\right)
$$

through creative applications of an integration method that we had previously introduced and applied to prove new Ramanujan-like formulas for $\frac{1}{\pi}$. We provide explicit closedform expressions for natural variants of the above series that cannot be evaluated by state-of-the-art computer algebra systems, such as the elegant symbolic evaluation

$$
\sum_{n=1}^{\infty} \frac{\left(\begin{array}{c}
2 n \\
n
\end{array}\right)^{2} H_{n}}{32^{n}(n+1)}=8-\frac{2 \Gamma\left(\frac{1}{4}\right)^{2}}{\pi^{3 / 2}}-\frac{4 \pi^{3 / 2}+16 \sqrt{\pi} \ln (2)}{\Gamma\left(\frac{1}{4}\right)^{2}}
$$

introduced in our present paper. We also discuss some related problems concerning binomial series containing alternating harmonic numbers. We also introduce a new class of harmonic summations for Catalan's constant $G$ and $\frac{1}{\pi}$ such as the series

$$
\sum_{n=1}^{\infty} \frac{\left(\begin{array}{c}
2 n \\
n
\end{array}\right)^{2} H_{n}}{16^{n}(n+1)^{2}}=16+\frac{32 G-64 \ln (2)}{\pi}-16 \ln (2)
$$

which we prove through a variation of our previous integration method for constructing $\frac{1}{\pi}$ series.

1991 AMS Mathematics subject classification. 33E20, 33B15.

Keywords and phrases. Harmonic number, Central binomial coefficient, Infinite series, Symbolic computation, Gamma function.

Received by the editors April 23, 2018. 
1. Introduction. In [6], it was noted that the elegant Ramanujanlike formula

$$
\sum_{n=1}^{\infty} \frac{\left(\begin{array}{c}
2 n \\
n
\end{array}\right)^{2} H_{n}}{16^{n}(2 n-1)^{2}}=\frac{12-16 \ln (2)}{\pi}
$$

may be proven through an application of a differential operator with respect to a parameter involved in a known hypergeometric identity; this technique is also used in [7] to prove (1.1). A similar strategy was also applied to prove an equivalent formulation of the known equation

$$
\sum_{n=1}^{\infty} \frac{\left(\begin{array}{c}
2 n \\
n
\end{array}\right)^{2} H_{n}}{(n+1) 16^{n}}=4-\frac{16 \ln (2)}{\pi}
$$

in 2017 in [11]. Both (1.1) and (1.2) are special cases of a very useful result introduced in [4] on the evaluation of series containing factors of the form $\left(\begin{array}{c}2 n \\ n\end{array}\right)^{2} H_{n}$ for indices $n \in \mathbb{N}$, which was proven through the use of a beta-like integral transform. While applying parameter derivatives to classical hypergeometric identities only produces specific results on harmonic summations containing squared central binomial coefficients as a factor in the summand, the integration technique presented in [4] may be applied much more generally. The power of the integration strategy considered in [4] motivates the exploration of further applications of the integral transform given in [4], as well as investigations on applications of analogues and variants of this integral transform.

On this latter note, a new integral transform $T_{\ln , \text { arcsin }}$ given in [5] in 2017 was used to evaluate new series for $\frac{1}{\pi}$ containing factors of the form $\left(\begin{array}{c}2 n \\ n\end{array}\right)^{2} H_{2 n}^{\prime}$ in [5], letting $H_{2 n}^{\prime}=1-\frac{1}{2}+\cdots-\frac{1}{2 n}$ denote the alternating harmonic number of order $2 n$ for $n \in \mathbb{N}$. The operator $T_{\ln , \arcsin }$, which is analogous to the integral transform introduced in [4], is used in [5] to prove the elegant formula

$$
\frac{4 G-12 \ln 2+6}{\pi}=\sum_{n=1}^{\infty} \frac{\left(\begin{array}{c}
2 n \\
n
\end{array}\right)^{2} H_{2 n}}{16^{n}(2 n-1)^{2}}
$$

for Catalan's constant $G$, which is also proven through the use of the generating function for $\left(C_{n} H_{2 n}^{\prime}: n \in \mathbb{N}_{0}\right)$ that was evaluated in [3], letting $C_{n}$ denote the $n^{\text {th }}$ entry in the Catalan sequence. As discussed 
in [4], it is not in general feasible to use known generating functions for sequences such as $\left(\left(\begin{array}{c}2 n \\ n\end{array}\right) H_{n}: n \in \mathbb{N}_{0}\right)$ to evaluate series with summands containing expressions of the form $\left(\begin{array}{c}2 n \\ n\end{array}\right)^{2} H_{n}$. In general, the evaluation of infinite series involving products of entries of the harmonic sequence and expressions such as $\left(\begin{array}{c}2 n \\ n\end{array}\right)^{2}$ is difficult, and often leads to surprising and elegant results in the field of classical analysis.

The problem of determining explicit symbolic evaluations for summations containing entries of harmonic-type sequences and central binomial coefficients is a deep and interesting subject that has been explored through the use of many different kinds of classical analysisbased techniques. In [9], a variety of infinite summations involving generalized harmonic numbers and central binomial coefficients are evaluated through the use of beta-like integrals. In [8], a more abstract way of "depicting" harmonic-like numbers is used, writing

$$
H_{n}=\sigma_{1}\left(1, \frac{1}{2}, \frac{1}{3}, \ldots, \frac{1}{n}\right)
$$

and

$$
\frac{H_{n}^{2}-H_{n}^{(2)}}{2}=\sigma_{2}\left(1, \frac{1}{2}, \frac{1}{3}, \ldots, \frac{1}{n}\right)
$$

letting

$$
\sigma_{m}\left(x_{1}, x_{2}, \ldots, x_{n}\right)=\sum_{1 \leq k_{1}<k_{2}<\cdots<k_{m} \leq n} x_{k_{1}} x_{k_{2}} \cdots x_{k_{m}}
$$

denote the elementary symmetric function of order $m$. The authors in [8] mainly explore the symbolic evaluation of infinite series involving central binomial coefficients as well as expressions such as $\sigma_{m}\left(1, \frac{1}{3^{2}}, \ldots, \frac{1}{(2 n-1)^{2}}\right)$ and $\sigma_{m}\left(1, \frac{1}{2^{2}}, \ldots, \frac{1}{(n-1)^{2}}\right)$. The results put forth in [8] are nicely representative of how mathematical problems concerning the symbolic computation of series involving harmonic-like numbers can be closely connected with seemingly unrelated subjects in the theory of symmetric functions and in number theory.

The series expansions for powers of the inverse sine mapping proven in [2] involve central binomial coefficients and "nested" harmonic-type 
multisums, including

$$
\sum_{n_{1}=1}^{k-1} \frac{1}{\left(2 n_{1}\right)^{2}} \sum_{n_{2}=1}^{n_{1}-1} \frac{1}{\left(2 n_{2}\right)^{2}} \cdots \sum_{n_{N}=1}^{n_{N-1}-1} \frac{1}{\left(2 n_{N}\right)^{2}} .
$$

Motivated in part by the main results from [2], and in particular the classical infinite series identity

$$
\frac{2}{3}\left(\arcsin \left(\frac{z}{2}\right)\right)^{4}=\sum_{n=1}^{\infty} \frac{H_{n-1}^{(2)}}{n^{2}\left(\begin{array}{c}
2 n \\
n
\end{array}\right)} z^{2 n}
$$

known to Ramanujan [1], the authors in [12] determine congruences for

$$
p \sum_{n=1}^{p-1} \frac{H_{n-1}(2)}{n^{d}\left(\begin{array}{c}
2 n \\
n
\end{array}\right)} t^{n} \quad(\bmod p)
$$

for prime numbers $p$ and for special values of $d$, generalizing congruence results given by Zhi-Wei Sun in [15], in which the elegant formula

$$
\frac{\pi^{3}}{48}=\sum_{n=1}^{\infty} \frac{2^{n} H_{n-1}^{(2)}}{n\left(\begin{array}{c}
2 n \\
n
\end{array}\right)}
$$

is also introduced. This discussion further illustrates how researching new kinds of subjects concerning summations containing harmoniclike numbers together with central binomial coefficients can lead to unexpected results in both applied analysis and number theory, and surprising connections between these disciplines.

In [10], new hypergeometric identities related to Ramanujan-like series for $\frac{1}{\pi}$ are proven using WZ-pairs, and an equivalent formulation of the beautiful Ramanujan-type [17] formula

$$
\sum_{n=1}^{\infty}\left(-\frac{1}{64}\right)^{n}\left(\begin{array}{c}
2 n \\
n
\end{array}\right)^{3}(4 n+1) H_{n}=\frac{2 \Gamma\left(\frac{9}{8}\right)^{2}}{3 \Gamma\left(\frac{7}{8}\right)^{2} \Gamma\left(\frac{5}{4}\right)^{2}}-\frac{4 \ln (2)}{\pi}
$$

is employed in the derivation of one of the main identities given in [10]. The related formula

$$
\sum_{n=0}^{\infty}\left(-\frac{1}{1024}\right)^{n}\left(\begin{array}{c}
2 n \\
n
\end{array}\right)^{5}\left(2-5(4 n+1) H_{n}\right)=\frac{4(5 \ln (2)-2 \pi)}{3 \Gamma\left(\frac{3}{4}\right)^{4}}
$$


was recently proven in [17] through the use of a parameter derivative applied to a classical ${ }_{6} F_{5}(-1)$ series identity. These striking results strongly inspire us to explore new techniques for computing series with summands with a factor of the form $H_{n}$ and fixed powers of $\left(\begin{array}{c}2 n \\ n\end{array}\right)$ in the numerator.

The beautiful formula

$$
\sum_{n=1}^{\infty}\left(\begin{array}{c}
2 n \\
n
\end{array}\right)^{3} \frac{H_{2 n}^{\prime}}{2^{8 n}}=\frac{\Gamma^{6}\left(\frac{1}{3}\right)(8 \sqrt{3} \ln 2-3 \pi)}{24 \cdot 2^{2 / 3} \pi^{4}}
$$

is proven in [18] through the use of special values of the multidimensional integral

$$
W_{n}(s):=\int_{[0,1]^{n}}\left|\sum_{k=1}^{n} e^{2 \pi x_{k} i}\right|^{s} d \mathbf{x},
$$

which, as discussed in [18], is used in the analysis of uniform planar random walks in the case whereby every step is a unit step, with the direction being random. In particular, the definite integral in (1.5) is equal to the $s^{\text {th }}$ moment of the distance in a given random walk, measured from the origin of the plane after a total of $n \in \mathbb{N}$ steps are taken. The delightful binomial-harmonic series given in (1.4) is proven through an identity for $W_{3}(s)$, which shows how series with binomial powers and entries in harmonic-like sequences can have direct applications in the theory of random walks, further motivating the exploration of new applications of the main techniques introduced in [4].

The conjectural equality

$$
\sum_{n=1}^{\infty} \frac{\left(\begin{array}{c}
2 n \\
n
\end{array}\right)^{2}}{n 16^{n}} H_{2 n}^{\prime}=\frac{2}{3} \sum_{n=0}^{\infty} \frac{\left(\begin{array}{c}
2 n \\
n
\end{array}\right)^{2} H_{2 n}}{(2 n+1) 16^{n}}
$$

was introduced in 2014 by Sun, with this conjecture being given alongside a number of conjectural $p$-adic congruences for truncated sums involving squared central binomial coefficients and harmonic numbers, in [14]. Considering the striking similarity between the righthand side of (1.6) and the series evaluated in (1.3), it is conceivable that the operator $T_{\ln , \text { arcsin }}$ and the integral transform from [4] could be used to prove the conjecture due to Sun in (1.6) as well as related results in the theory of $p$-adic congruences. Sun also introduced a 
plethora of conjectural formulas for infinite sums involving harmonic numbers and centered binomial coefficients in [16]. From the preceding discussion, we are highly motivated to pursue research endeavors on further applications of the integration methods from [4] and [5].

The application of parameter derivatives to hypergeometric identities to prove new results on binomial series containing harmonic numbers was recently discussed in [13]. In [13], formulas for evaluating harmonic summations of the following forms are proven using this method.

$$
\begin{aligned}
& \sum_{n=1}^{\infty} \frac{(2 a)_{n}(1-2 a)_{n}}{(n !)^{2}} \frac{H_{n}}{2^{n}} \\
& \sum_{n=1}^{\infty} \frac{(2 a)_{n}(2 b)_{n}}{n !\left(a+b+\frac{1}{2}\right)_{n}} \frac{H_{n}}{n+1}
\end{aligned}
$$

Letting the parameter $a$ given in the former series be equal to $\frac{1}{4}$, we obtain the series

$$
\sum_{n=1}^{\infty}\left(\begin{array}{c}
2 n \\
n
\end{array}\right)^{2} \frac{H_{n}}{32^{n}}
$$

which is evaluated in terms of the gamma function in [13]. Since the integration technique introduced in [4] is very useful for evaluating many different kinds of summations involving factors of the form $\left(\begin{array}{c}2 n \\ n\end{array}\right)^{2} H_{n}$, whereas the summation techniques considered [13] are given by differentiating specific hypergeometric identities, it is natural to consider the problem of evaluating generalizations of the series in (1.7), through the use of the integration method given in [4], which is described in Section 1.1. In particular, for a rational function $r(n)$, it is not obvious as to how to compute generalizations of (1.7) of the form

$$
\sum_{n=1}^{\infty}\left(\begin{array}{c}
2 n \\
n
\end{array}\right)^{2} \frac{H_{n} \cdot r(n)}{32^{n}}
$$

following the strategies outlined in [13], or using the generating functions for sequences involving products of harmonic numbers and central binomial coefficients given in [3].

The infinite series in (1.7) also recently appeared in [17]. In [17], it 
is noted that by Bailey's theorem, we have that

$$
{ }_{2} F_{1}\left[\begin{array}{c|c}
a, 1-a & \frac{1}{2} \\
c & 2
\end{array}\right]=\frac{\Gamma\left(\frac{c}{2}\right) \Gamma\left(\frac{c+1}{2}\right)}{\Gamma\left(\frac{a+c}{2}\right) \Gamma\left(\frac{1-a+c}{2}\right)},
$$

and that (1.7) may be computed in terms of the gamma function by applying the operator $\frac{\partial}{\partial c}$ to both sides of (1.9) in the case whereby $a=\frac{1}{2}$. A formula for a $p$-adic analogue of (1.7) is also proven in [17], and many supercongruences for finite sums with central binomial coefficients and harmonic-type numbers are established. The lovely formula

$$
\sum_{n=1}^{\infty}\left(\begin{array}{c}
2 n \\
n
\end{array}\right)^{3} \frac{H_{n}}{64^{n}}=\frac{2 \pi(\pi-3 \ln 2)}{3 \Gamma^{4}\left(\frac{3}{4}\right)}
$$

is also proven in [17] following the "usual" method of applying a parameter derivative to both sides of a known hypergeometric identity; in this case, the classical result known as Dixon's theorem is used. Instead of applying partial derivative operators to classical hypergeometric series identities, in our present article we make use of something of an inverse approach by showing how the integration method explored in [4] can be used in a very general way to evaluate series involving harmonic numbers and squared central binomial coefficients.

From the above discussion, we see that the problem of determining closed-form expressions for harmonic summations of the form in (1.8) is an intriguing subject with many potential applications. In our present article, we offer vast generalizations of the formula

$$
\sum_{n=1}^{\infty}\left(\begin{array}{c}
2 n \\
n
\end{array}\right)^{2} \frac{H_{n}}{32^{n}}=\frac{\sqrt{\pi}(\pi-4 \ln 2)}{2 \Gamma^{2}\left(\frac{3}{4}\right)}
$$

that had been noted by Tauraso in 2017 in [17] and by Nicholson in 2018 in [13] by showing how creative applications of the integration strategy we had previously introduced in [4] can be used to evaluate series of the following forms for $z \in \mathbb{Z}_{>0}$.

$$
\sum_{n \in \mathbb{N}} \frac{\left(\begin{array}{c}
2 n \\
n
\end{array}\right)^{2} H_{n}}{32^{n}(n+z)} \quad \sum_{n \in \mathbb{N}} \frac{\left(\begin{array}{c}
2 n \\
n
\end{array}\right)^{2} H_{n}}{32^{n}(2 n-2 z+1)} \quad \sum_{n \in \mathbb{N}} \frac{\left(\begin{array}{c}
2 n \\
n
\end{array}\right)^{2} H_{n}}{32^{n}(2 n-2 z+1)^{2}}
$$

In Section 1.1, we briefly review some preliminary results that we need for the main proofs in our article. In Section 2, we offer a new 
proof of the evaluation for (1.7) using the integral transform from [4], to illustrate the idea of applying this integration technique with respect to summations of the form given in (1.8), and we prove the following new formulas.

$$
\begin{aligned}
& \sum_{n=1}^{\infty}\left(\frac{1}{32}\right)^{n} \frac{\left(\begin{array}{c}
2 n \\
n
\end{array}\right)^{2} H_{n}}{n+1}=8-\frac{2 \Gamma\left(\frac{1}{4}\right)^{2}}{\pi^{3 / 2}}-\frac{4 \pi^{3 / 2}+16 \sqrt{\pi} \ln (2)}{\Gamma\left(\frac{1}{4}\right)^{2}} \\
& \sum_{n=1}^{\infty}\left(\frac{1}{32}\right)^{n} \frac{\left(\begin{array}{c}
2 n \\
n
\end{array}\right)^{2} H_{n}}{2 n-1}= \\
& \frac{2 \Gamma\left(\frac{5}{4}\right)^{2}(4 \ln (2)-\pi)}{\pi^{3 / 2}}+\frac{\Gamma\left(\frac{3}{4}\right)(4 \ln (2)+\pi-4)}{\sqrt{2 \pi} \Gamma\left(\frac{1}{4}\right)} \\
& \sum_{n=1}^{\infty}\left(\frac{1}{32}\right)^{n} \frac{\left(\begin{array}{c}
2 n \\
n
\end{array}\right)^{2} H_{n}}{(2 n-1)^{2}}= \\
& \frac{(\pi-4 \ln (2)) \Gamma\left(\frac{1}{4}\right)^{2}}{8 \pi^{3 / 2}}-\sqrt{\frac{2}{\pi}} \frac{(\pi+4 \ln (2)-6) \Gamma\left(\frac{3}{4}\right)}{\Gamma\left(\frac{1}{4}\right)} .
\end{aligned}
$$

In Section 3, we show how the proof techniques applied in Section 2 can be generalized, and in Section 4 we introduce a new class of Ramanujan-like series for $\frac{1}{\pi}$ using a proof technique from Section 3 . Finally, in Section 5, we discuss some future avenues of research related to the main results in our present article.

1.1. Preliminaries. The equality whereby

$$
2 \sum_{n=1}^{\infty} \frac{(2 a)_{n}(2 b)_{n}}{n !\left(a+b+\frac{1}{2}\right)_{n}} \frac{H_{n}}{2^{n}}=\sum_{n=1}^{\infty} \frac{(a)_{n}(b)_{n}}{n !\left(a+b+\frac{1}{2}\right)_{n}} H_{n},
$$

is proven in [13], for elements $a$ and $b$ in the set of complex numbers such that $a+b-\frac{1}{2} \notin \mathbb{Z}_{<0}$, and this identity is used to prove that the series $\sum_{n=1}^{\infty} \frac{(2 a)_{n}(1-2 a)_{n}}{(n !)^{2}} \frac{H_{n}}{2^{n}}$ is equal to

$$
\frac{\sqrt{\pi}}{2 \Gamma(1-a) \Gamma\left(a+\frac{1}{2}\right)}\left(\psi(1-a)+\psi\left(a+\frac{1}{2}\right)-\psi(1)-\psi\left(\frac{1}{2}\right)\right),
$$


letting $\psi(x)=\frac{\Gamma^{\prime}(x)}{\Gamma(x)}$ denote the digamma function, thus leading to the evaluation of (1.7) noted in Theorem 2.1 below. We present a generalization of this theorem in our present article, using the main method applied in [4], which may be summarized in Lemma 1.1 below.

As noted above, the elegant formula in (1.10), which has motivated much of the work put forth in our present paper, also appears in [17] and is proven through a straightforward application of Bailey's theorem. We offer a new proof of (1.10) that is significantly different compared to the proofs of this result from both [17] and [13]. Variants of our proof of (1.10) may be used to greatly generalize the formula in (1.10) through the use of the following fundamental lemma from [4], as we later discuss.

Lemma 1.1. Letting $f: \mathbb{N}_{0} \rightarrow \mathbb{C}$ and $g(n)=\frac{f(n)}{16^{n}(2 n-1)}$, if $f$ is such that

$$
\sum_{n=0}^{\infty}(-1)^{n}\left(\begin{array}{c}
\frac{1}{2} \\
n
\end{array}\right) f(n) \frac{x^{2 n} \ln \left(1-x^{2}\right)}{\sqrt{1-x^{2}}}
$$

is integrable on $[0,1]$, it follows that

$$
\sum_{n=0}^{\infty} g(n)\left(\begin{array}{c}
2 n \\
n
\end{array}\right)^{2} H_{n}
$$

is equal to $\frac{2}{\pi}$ times the sum of

$$
\int_{0}^{1}\left(\sum_{n=0}^{\infty}(-1)^{n}\left(\begin{array}{c}
\frac{1}{2} \\
n
\end{array}\right) f(n) \frac{x^{2 n} \ln \left(1-x^{2}\right)}{\sqrt{1-x^{2}}}\right) d x
$$

and $\pi \ln (2) \sum_{n=0}^{\infty} \frac{\left(\begin{array}{c}2 n \\ n\end{array}\right)^{2} f(n)}{16^{n}(1-2 n)}$.

The above lemma often allows us to express an infinite series of the form

$$
\sum_{n=1}^{\infty} g(n)\left(\begin{array}{c}
2 n \\
n
\end{array}\right)^{2} H_{n}
$$

in a convenient way in terms of a relatively "manageable" definite integral over an elementary function. As discussed in [4], this is very useful because it is not obvious in general how to symbolically compute 
series of the form noted in (1.12) by applying parameter derivatives to known hypergeometric identities.

We remark that throughout the course of our present article, expressions such as "closed form" are meant to include evaluations involving the gamma function. Also, it is useful to note that it is convenient for our purposes to use the "Mathematica definition" of the complete elliptic integral of the first kind $\mathbf{K}$ whereby

$$
\mathbf{K}(k)=\frac{\pi}{2} \cdot{ }_{2} F_{1}\left[\begin{array}{c|c}
\frac{1}{2}, \frac{1}{2} & k \\
1 & k
\end{array}\right]
$$

with $\mathbf{E}$ defined so that

$$
\mathbf{E}(k)=\frac{\pi}{2} \cdot{ }_{2} F_{1}\left[\begin{array}{c|c}
-\frac{1}{2}, \frac{1}{2} & k \\
1 &
\end{array}\right] .
$$

2. Motivating examples. As we previously noted, the following theorem follows immediately from the formula for (1.11) given in [13]. We offer a new proof of this result, to illustrate how the main technique in [4] can be used to evaluate series as in (1.8).

Theorem 2.1. $\sum_{n=1}^{\infty}\left(\begin{array}{c}2 n \\ n\end{array}\right)^{2} \frac{H_{n}}{32^{n}}=\frac{\Gamma^{2}\left(\frac{1}{4}\right)}{4 \sqrt{\pi}}\left(1-\frac{4 \ln (2)}{\pi}\right)[\mathbf{1 3}]$.

Proof. Letting $f(n)$ be equal to $2^{-n}(2 n-1)$ in Lemma 1.1, we find that the series given in the above theorem is equal to:

$$
-\frac{2 \sqrt{2}}{\pi} \int_{0}^{1} \frac{\ln \left(1-x^{2}\right)}{\sqrt{1-x^{2}} \sqrt{2-x^{2}}} d x-\frac{\Gamma\left(\frac{1}{4}\right)^{2} \ln (2)}{\pi^{3 / 2}} .
$$

Using the substitution $u=1-x^{2}$ in the above integrand, we find that

$$
\sum_{n=1}^{\infty}\left(\begin{array}{c}
2 n \\
n
\end{array}\right)^{2} \frac{H_{n}}{32^{n}}=-\frac{\sqrt{2}}{\pi} \int_{0}^{1} \frac{\ln (u)}{\sqrt{1-u^{2}} \sqrt{u}} d u-\frac{\Gamma\left(\frac{1}{4}\right)^{2} \ln (2)}{\pi^{3 / 2}}
$$

The Mathematica computer algebra system is able to evaluate the above integral directly, yielding the desired result. Alternatively, the substitution of the Maclaurin series for $\frac{1}{\sqrt{1-u^{2}}}$ in the above integrand also may be used to symbolically compute the above integral. 
To show how Lemma 1.1 may be applied in a non-trivial way to evaluate classes of variants of the summation given in Theorem 2.1, we consider the problem of evaluating the following natural analogue of the harmonic summation given in the above theorem.

$$
\sum_{n=1}^{\infty} \frac{\left(\begin{array}{c}
2 n \\
n
\end{array}\right)^{2} H_{n}}{32^{n}(n+1)}
$$

It is not obvious as to how to evaluate this series following the techniques given in [13]. Mathematica 11 is not able to provide any kind of closed-form evaluation for the series in (2.1), and it is not obvious as to how to apply known integral formulas for harmonic numbers to evaluate this sum. For example, through an application of the formula

$$
\int_{0}^{1} \frac{1-x^{n}}{1-x} d x=H_{n}
$$

we see that the summation in (2.1) may be expressed as

$$
\int_{0}^{1} \frac{\frac{8 \mathbf{E}\left(\frac{x}{2}\right)+4(x-2) \mathbf{K}\left(\frac{x}{2}\right)}{\pi x}-\frac{\sqrt{\frac{2}{\pi}} \Gamma\left(\frac{3}{4}\right)}{\Gamma\left(\frac{5}{4}\right)}}{x-1} d x,
$$

but it is not at all obvious how to compute the difficult integral given above. Similarly, through the use of the integral identity whereby

$$
H_{n}=-n \cdot \int_{x \in[0,1]} \ln (1-x) \cdot x^{n-1} d x
$$

for $n \in \mathbb{N}_{0}$, we find that the problem of symbolically computing (2.1) is equivalent to the difficult problem of evaluating the following integrals.

$$
\begin{aligned}
& -\frac{1}{16} \int_{0}^{1}{ }_{2} F_{1}\left[\begin{array}{c|c}
\frac{3}{2}, \frac{3}{2} & x \\
3 & \frac{2}{2}
\end{array}\right] \ln (1-x) d x= \\
& \frac{4}{\pi} \int_{0}^{1}\left(\frac{\mathbf{E}\left(\frac{x}{2}\right)}{x^{2}}+\frac{\left(\frac{x}{2}-2\right) \mathbf{K}\left(\frac{x}{2}\right)}{x^{2}}\right) \ln (1-x) d x
\end{aligned}
$$

In a similar fashion, it appears that it would be infeasible to make use of known integral formulas for central binomial coefficients or Catalan-type numbers to determine a closed-form expression for the 
infinite series in (2.1). To illustrate this assertion, if we factor out the expression $C_{n}=\frac{1}{n+1}\left(\begin{array}{c}2 n \\ n\end{array}\right)$ in the summand in this series and replace this factor with a standard integral expression for the Catalan number $C_{n}$ of order $n$, we see that the infinite sum in (2.1) may be expressed as

$$
\frac{2 \sqrt{2}}{\pi} \int_{0}^{4} \sqrt{\frac{4-x}{x(8-x)}} \ln \left(\frac{1}{2}+\sqrt{\frac{2}{8-x}}\right) d x
$$

which cannot be evaluated by state-of-the-art CAS programs. Similarly, if we substitute a Wallis-type integral into the summand in (2.1), this would yield a very recalcitrant integral such as that given below.

$$
\begin{aligned}
& \frac{1}{\pi} \int_{0}^{2 \pi}\left(4 \operatorname { s e c } ^ { 2 } ( t ) \left(\sqrt{1-\frac{\cos ^{2}(t)}{2}} \ln \left(2 \sqrt{1-\frac{\cos ^{2}(t)}{2}}\right)-\right.\right. \\
& \left.\left.\left(\sqrt{1-\frac{\cos ^{2}(t)}{2}}+1\right) \ln \left(\sqrt{1-\frac{\cos ^{2}(t)}{2}}+1\right)+\ln (2)\right)\right) d t
\end{aligned}
$$

The above discussion shows that it is not feasible to use standard or conventional integration methods to evaluate

$$
\sum_{n=1}^{\infty} \frac{\left(\begin{array}{c}
2 n \\
n
\end{array}\right)^{2} H_{n}}{32^{n}(n+1)}
$$

which shows, in part, why determining the closed-form evaluation given below is challenging. Our proof of the following theorem nicely illustrates how the technique introduced in [4] can be applied in a creative way to produce a simple closed-form evaluation that does not follow immediately from Lemma 1.1 .

Theorem 2.2. The series

$$
\sum_{n=1}^{\infty}\left(\frac{1}{32}\right)^{n} \frac{\left(\begin{array}{c}
2 n \\
n
\end{array}\right)^{2} H_{n}}{n+1}
$$

is equal to

$$
8-\frac{2 \Gamma\left(\frac{1}{4}\right)^{2}}{\pi^{3 / 2}}-\frac{4 \pi^{3 / 2}+16 \sqrt{\pi} \ln (2)}{\Gamma\left(\frac{1}{4}\right)^{2}}
$$


Proof. Letting $f(n)=\left(\frac{1}{2}\right)^{n} \frac{2 n-1}{n+1}$, by Lemma 1.1, we have that the series $\sum_{n=0}^{\infty}\left(\frac{1}{32}\right)^{n} \frac{\left(\begin{array}{c}2 n \\ n\end{array}\right)^{2} H_{n}}{n+1}$ is equal to the following expression:

$$
\frac{4}{\pi} \int_{0}^{1} \frac{\left(\sqrt{4-2 x^{2}}-2\right) \ln \left(1-x^{2}\right)}{x^{2} \sqrt{1-x^{2}}} d x-\frac{16 \sqrt{\pi} \ln (2)}{\Gamma\left(\frac{1}{4}\right)^{2}} .
$$

So, the problem of computing the series given in Theorem 2.2 is equivalent to the problem of evaluating the following integrals, neither of which can be directly evaluated in closed form by state-of-the-art computer algebra systems.

$$
\int_{0}^{1} \frac{\left(\sqrt{4-2 x^{2}}-2\right) \ln \left(1-x^{2}\right)}{x^{2} \sqrt{1-x^{2}}} d x=\int_{0}^{1} \frac{(\sqrt{2} \sqrt{1+u}-2) \ln (u)}{2(1-u)^{3 / 2} \sqrt{u}} d u
$$

Rewriting the expression

$$
\int_{0}^{1} \frac{(-2+\sqrt{2} \sqrt{1+u}) \ln (u)}{(1-u)^{3 / 2} \sqrt{u}} d u
$$

as

$$
4 \pi+\sqrt{2} \int_{0}^{1} \frac{\sqrt{1+u} \ln (u)}{(1-u)^{3 / 2} \sqrt{u}} d u
$$

we find that current CAS software also cannot compute this latter integral.

Our strategy for computing (2.2) in closed form is to: find a formula for the Maclaurin series coefficients for the expression $\frac{\sqrt{1+u}}{(1-u)^{3 / 2}}$ in the integrand in (2.2), then multiply each term in the corresponding series expansion by $\frac{\ln (u)}{\sqrt{u}}$, and then integrate term-by-term. This may appear to be a very roundabout way of determining the symbolic value of the definite integral in (2.2), but it is not at all clear what kinds of integration methods could be successfully applied to find (2.2) in closed form.

Since

$$
\frac{\sqrt{1+u}}{(1-u)^{3 / 2}}=\sum_{n=0}^{\infty} \frac{u^{2 n}(1+2 u+4 n(1+u)) \Gamma\left(\frac{1}{2}+n\right)}{\sqrt{\pi} \Gamma(1+n)}
$$


we have that

$$
\frac{\sqrt{1+u}}{(1-u)^{3 / 2}}=\sum_{n=0}^{\infty} \frac{(1+4 n) u^{2 n} \Gamma\left(\frac{1}{2}+n\right)}{\sqrt{\pi} \Gamma(1+n)}+\sum_{n=0}^{\infty} \frac{4 u^{1+2 n} \Gamma\left(\frac{3}{2}+n\right)}{\sqrt{\pi} \Gamma(1+n)}
$$

so that we may evaluate the definite integral in (2.2) explicitly as follows:

$$
\begin{aligned}
& \int_{0}^{1} \frac{\sqrt{1+u} \ln (u)}{(1-u)^{3 / 2} \sqrt{u}} d u=\sum_{n=0}^{\infty} \frac{(1+4 n)(-4) \Gamma\left(\frac{1}{2}+n\right)}{(1+4 n)^{2}(\sqrt{\pi} \Gamma(1+n))}+ \\
& \sum_{n=0}^{\infty} \frac{4(-4) \Gamma\left(\frac{3}{2}+n\right)}{(3+4 n)^{2}(\sqrt{\pi} \Gamma(1+n))} \\
& \\
&=-\frac{\Gamma\left(\frac{1}{4}\right)^{2}+\pi \Gamma\left(\frac{3}{4}\right)^{2}}{\sqrt{2 \pi}} .
\end{aligned}
$$

We thus obtain the desired result.

As discussed in the Introduction, the integration strategy given in [4] is much more versatile compared to the use of specific hypergeometric identities to produce specific results on harmonic summations. To further illustrate this idea, we offer a complete proof of the following new evaluation.

Theorem 2.3. The following equality holds:

$$
\sum_{n=1}^{\infty} \frac{\left(\begin{array}{c}
2 n \\
n
\end{array}\right)^{2} H_{n}}{32^{n}(2 n-1)}=\frac{2 \Gamma\left(\frac{5}{4}\right)^{2}(4 \ln (2)-\pi)}{\pi^{3 / 2}}+\frac{\Gamma\left(\frac{3}{4}\right)(4 \ln (2)+\pi-4)}{\sqrt{2 \pi} \Gamma\left(\frac{1}{4}\right)} .
$$

Proof. We observe that

$$
\sum_{n=0}^{\infty} \frac{32^{-n}\left(\begin{array}{c}
2 n \\
n
\end{array}\right)^{2} H_{n}}{2 n-1}
$$

is equal to

$$
\frac{2\left(\int_{0}^{1} \frac{\sqrt{2-x^{2}} \ln \left(1-x^{2}\right)}{\sqrt{2} \sqrt{1-x^{2}}} d x+\pi \ln (2)\left(\frac{2 \sqrt{\pi}}{\Gamma\left(\frac{1}{4}\right)^{2}}+\frac{4 \Gamma\left(\frac{5}{4}\right)^{2}}{\pi^{3 / 2}}\right)\right)}{\pi}
$$


from the main lemma given in [4], and we thus observe that the series given in Theorem 2.3 is also equal to

$$
\frac{2\left(\frac{\int_{0}^{1} \frac{\sqrt{1+u} \ln (u)}{\sqrt{1-u} \sqrt{u}} d u}{2 \sqrt{2}}+\pi \ln (2)\left(\frac{2 \sqrt{\pi}}{\Gamma\left(\frac{1}{4}\right)^{2}}+\frac{4 \Gamma\left(\frac{5}{4}\right)^{2}}{\pi^{3 / 2}}\right)\right)}{\pi} .
$$

The Mathematica computer algebra system is able to directly evaluate the datum from (2.5), yielding the desired result.

Theorem 2.4. The series

$$
\sum_{n=1}^{\infty}\left(\frac{1}{32}\right)^{n} \frac{\left(\begin{array}{c}
2 n \\
n
\end{array}\right)^{2} H_{n}}{(2 n-1)^{2}}
$$

is equal to

$$
\frac{(\pi-4 \ln (2)) \Gamma\left(\frac{1}{4}\right)^{2}}{8 \pi^{3 / 2}}-\sqrt{\frac{2}{\pi}} \frac{(\pi+4 \ln (2)-6) \Gamma\left(\frac{3}{4}\right)}{\Gamma\left(\frac{1}{4}\right)} .
$$

Proof. We begin by noting that a direct application of the fundamental lemma from [4] shows that the series given in the above theorem, which cannot be evaluated using computer algebra systems such as Mathematica 11, is equal to the following expression.

$$
\begin{aligned}
& -\frac{\sqrt{2}}{\pi} \int_{0}^{1} \frac{\left(\sqrt{2-x^{2}}+x \sin ^{-1}\left(\frac{x}{\sqrt{2}}\right)\right) \ln \left(1-x^{2}\right)}{\sqrt{1-x^{2}}} d x \\
& -\frac{\sqrt{\frac{2}{\pi}}\left(\Gamma\left(\frac{3}{4}\right)^{2}+2 \Gamma\left(\frac{5}{4}\right)^{2}\right) \ln (2)}{\Gamma\left(\frac{3}{4}\right) \Gamma\left(\frac{5}{4}\right)}
\end{aligned}
$$

Expanding the above integrand, we again encounter the integral

$$
\int_{0}^{1} \frac{\sqrt{2-x^{2}} \ln \left(1-x^{2}\right)}{\sqrt{1-x^{2}}} d x
$$

which we had previously seen in (2.4). So, we find that the infinite sum given in Theorem 2.4 is also equal to the following.

$$
-\frac{\sqrt{2}}{\pi} \int_{0}^{1} \frac{x \sin ^{-1}\left(\frac{x}{\sqrt{2}}\right) \ln \left(1-x^{2}\right)}{\sqrt{1-x^{2}}} d x
$$




$$
+\frac{\Gamma\left(\frac{1}{4}\right)(\pi-4 \ln (2))}{4 \sqrt{2 \pi} \Gamma\left(\frac{3}{4}\right)}-\frac{\Gamma\left(\frac{3}{4}\right)^{2}(\pi+8 \ln (2)-4)}{2 \pi^{3 / 2}}
$$

By rewriting the above integral as

$$
\int_{0}^{1} \frac{\sin ^{-1}\left(\frac{\sqrt{1-u}}{\sqrt{2}}\right) \ln (u)}{2 \sqrt{u}} d u
$$

and then substituting the expression $\frac{\sqrt{1-u}}{\sqrt{2}}$ into the Maclaurin series for the inverse sine, we see that the definite integral given in (2.6) is also equal to

$$
-2^{-\frac{5}{2}} \pi \sum_{n=0}^{\infty} \frac{2^{-5 n}\left(\begin{array}{c}
2 n \\
n
\end{array}\right)^{2} H_{n+1}}{n+1}-\frac{\sqrt{\pi} \Gamma\left(\frac{3}{4}\right) \ln (4)}{4 \Gamma\left(\frac{5}{4}\right)}
$$

and we may thus apply Theorem 2.2 to yield the desired result.

3. Generalizations and variations. We begin by noting that one of the key ingredients in our proof of Theorem 2.2 was based on the the use of a Maclaurin-type series for the expression

$$
\frac{\sqrt{1+u}}{(1-u)^{3 / 2}}
$$

for $u \in[0,1)$, and that the determination of a suitable power series expansion for this expression was nontrivial in that it is not obvious as to how to find explicit formulas for the Taylor series coefficients for (3.1) without already knowing the formula we had introduced in (2.3). For our strategy in computing the Maclaurin series coefficients for (3.1), we had planned to make use of known results on the series expansion for

$$
\sqrt{\frac{1+u}{1-u}}
$$

and then "interpret" the left-hand factor in the left-hand side of

$$
\frac{1}{1-u} \sqrt{\frac{1+u}{1-u}}=\frac{\sqrt{1+u}}{(1-u)^{3 / 2}}
$$

as a partial sum operator. The preceding discussion nicely illustrates how successful applications of the fundamental lemma from [4] often require creative manipulations of generating functions. 
It seems that applying the proof technique for Theorem 2.2 to try to determine the value of

$$
\sum_{n=1}^{\infty} \frac{\left(\begin{array}{c}
2 n \\
n
\end{array}\right)^{2} H_{n}}{32^{n}(n+2)}
$$

may be cumbersome or infeasible. However, by means of a simple re-indexing argument, we see that there is a very elegant connection between (3.2) and Theorem 2.2, Theorem 2.3, and Theorem 2.4.

Theorem 3.1. The series

$$
\sum_{n=1}^{\infty} \frac{\left(\begin{array}{c}
2 n \\
n
\end{array}\right)^{2} H_{n}}{32^{n}(n+2)}
$$

is equal to

$$
\frac{64}{9}-\frac{\Gamma\left(\frac{1}{4}\right)^{2}(\pi-4 \ln (2)+18)}{9 \pi^{3 / 2}}-\frac{2 \sqrt{\frac{2}{\pi}} \Gamma\left(\frac{3}{4}\right)(9 \pi+36 \ln (2)-16)}{9 \Gamma\left(\frac{1}{4}\right)} .
$$

Proof. Apply the re-indexing technique outlined below.

$$
\begin{aligned}
& \sum_{n=1}^{\infty} \frac{\left(\begin{array}{c}
2 n \\
n
\end{array}\right)^{2} H_{n}}{32^{n}(n+2)} \\
& =\sum_{n=2}^{\infty}\left(\frac{1}{32}\right)^{n-1} \frac{\left(\begin{array}{c}
2 n-2 \\
n-1
\end{array}\right)^{2} H_{n-1}}{n+1} \\
& =\sum_{n=2}^{\infty}\left(\frac{1}{32}\right)^{n-1} \frac{\left(\begin{array}{c}
2 n-2 \\
n-1
\end{array}\right)^{2}\left(H_{n}-\frac{1}{n}\right)}{n+1} \\
& =\sum_{n=2}^{\infty}\left(\frac{32^{1-n}\left(\begin{array}{c}
2 n-2 \\
n-1
\end{array}\right)^{2} H_{n}}{n+1}-\frac{32^{1-n}\left(\begin{array}{c}
2 n-2 \\
n-1
\end{array}\right)^{2}}{n(n+1)}\right) \\
& =-\frac{\sqrt{\frac{2}{\pi}} \Gamma\left(\frac{1}{4}\right)-3 \Gamma\left(\frac{7}{4}\right)}{6 \Gamma\left(\frac{7}{4}\right)}+32 \sum_{n=2}^{\infty} \frac{32^{-n}\left(\begin{array}{c}
2 n-2 \\
n-1
\end{array}\right) H_{n}^{2}}{n+1} \\
& =-\frac{\sqrt{\frac{2}{\pi}} \Gamma\left(\frac{1}{4}\right)-3 \Gamma\left(\frac{7}{4}\right)}{6 \Gamma\left(\frac{7}{4}\right)}+8 \sum_{n=2}^{\infty} \frac{2^{-5 n} n^{2}\left(\begin{array}{c}
2 n \\
n
\end{array}\right)^{2} H_{n}}{(n+1)(2 n-1)^{2}}
\end{aligned}
$$




$$
\begin{aligned}
& =-\frac{\sqrt{\frac{2}{\pi}} \Gamma\left(\frac{1}{4}\right)-3 \Gamma\left(\frac{7}{4}\right)}{6 \Gamma\left(\frac{7}{4}\right)}+ \\
& 8 \sum_{n=2}^{\infty} 2^{-5 n}\left(\begin{array}{c}
2 n \\
n
\end{array}\right)^{2} H_{n}\left(\frac{1}{9(n+1)}+\frac{1}{6(2 n-1)^{2}}+\frac{5}{18(2 n-1)}\right)
\end{aligned}
$$

We now have that the desired result follows immediately from Theorem 2.2, Theorem 2.3, and Theorem 2.4.

To compute a given series of the form

$$
\sum_{n=1}^{\infty} \frac{\left(\begin{array}{c}
2 n \\
n
\end{array}\right)^{2} H_{n}}{32^{n}(n+z)}
$$

in closed form for $z \in \mathbb{Z}_{>0}$, we make use of the inductive approach described below. We begin by re-writing the above summation as suggested below.

$$
\begin{aligned}
& \sum_{n=1}^{\infty} \frac{\left(\begin{array}{c}
2 n \\
n
\end{array}\right)^{2} H_{n}}{32^{n}(n+z)} \\
& =\sum_{n=1}^{\infty} \frac{\left(\begin{array}{c}
2 n \\
n
\end{array}\right)^{2} H_{n}}{32^{n}((n+1)+z-1)} \\
& =\sum_{n=2}^{\infty} \frac{\left(\begin{array}{c}
2 n-2 \\
n-1
\end{array}\right)^{2} H_{n-1}}{32^{n-1}(n+z-1)} \\
& =8 \sum_{n=2}^{\infty} \frac{2^{-5 n} n^{2}\left(\begin{array}{c}
2 n \\
n
\end{array}\right)^{2} H_{n-1}}{(2 n-1)^{2}(n+z-1)} \\
& =8 \sum_{n=2}^{\infty} \frac{2^{-5 n} n^{2}\left(\begin{array}{c}
2 n \\
n
\end{array}\right)^{2}\left(H_{n}-\frac{1}{n}\right)}{(2 n-1)^{2}(n+z-1)} \\
& =8 \sum_{n=2}^{\infty} \frac{2^{-5 n} n^{2}\left(\begin{array}{c}
2 n \\
n
\end{array}\right)^{2} H_{n}}{(2 n-1)^{2}(n+z-1)}-8 \sum_{n=2}^{\infty} \frac{2^{-5 n} n\left(\begin{array}{c}
2 n \\
n
\end{array}\right)^{2}}{(2 n-1)^{2}(n+z-1)}
\end{aligned}
$$

It is easily seen that hypergeometric series of the form

$$
\sum_{n}\left(\frac{1}{32}\right)^{n} \frac{n\left(\begin{array}{c}
2 n \\
n
\end{array}\right)^{2}}{(2 n-1)^{2}(n+m)}
$$


always have closed-form evaluations for $m \in \mathbb{N}$, as may be verified by writing

$$
\sum_{n=0}^{\infty} \frac{n\left(\begin{array}{c}
2 n \\
n
\end{array}\right)^{2} x^{n+m-1}}{32^{n}(2 n-1)^{2}}=\frac{1}{8} x^{m}{ }_{2} F_{1}\left[\begin{array}{c|c}
\frac{1}{2}, \frac{1}{2} & \frac{x}{2} \\
2 &
\end{array}\right]
$$

and evaluating the above expressions as

$$
\frac{1}{8} x^{m}\left(\frac{4\left(1-\frac{2}{x}\right) \mathbf{K}\left(\frac{x}{2}\right)}{\pi}+\frac{8 \mathbf{E}\left(\frac{x}{2}\right)}{\pi x}\right)
$$

and then using known results on moments of complete elliptic integrals. Now, we observe that we may expand the factor

$$
\frac{n^{2}}{(2 n-1)^{2}(n+z-1)}
$$

from the summand in the series

$$
\sum_{n} \frac{n^{2}}{(2 n-1)^{2}(n+z-1)}\left(\frac{1}{32}\right)^{n}\left(\begin{array}{c}
2 n \\
n
\end{array}\right)^{2} H_{n}
$$

as follows.

$$
\begin{aligned}
& \frac{n^{2}}{(2 n-1)^{2}(n+z-1)}= \\
& \left(\frac{z-1}{2 z-1}\right)^{2} \cdot \frac{1}{n+z-1}+ \\
& \frac{4 z-3}{2(2 z-1)^{2}} \cdot \frac{1}{2 n-1}+ \\
& \frac{1}{2(2 z-1)} \cdot \frac{1}{(2 n-1)^{2}}
\end{aligned}
$$

So, in our attempts to compute

$$
\sum_{n=1}^{\infty} \frac{\left(\begin{array}{c}
2 n \\
n
\end{array}\right)^{2} H_{n}}{32^{n}(n+z)}
$$

in closed form, we see that this problem amounts to the symbolic computation of the following series.

$$
\sum_{n=1}^{\infty} \frac{\left(\begin{array}{c}
2 n \\
n
\end{array}\right)^{2} H_{n}}{32^{n}(n+(z-1))} \quad \sum_{n=1}^{\infty} \frac{\left(\begin{array}{c}
2 n \\
n
\end{array}\right)^{2} H_{n}}{32^{n}(2 n-1)} \quad \sum_{n=1}^{\infty} \frac{\left(\begin{array}{c}
2 n \\
n
\end{array}\right)^{2} H_{n}}{32^{n}(2 n-1)^{2}}
$$


So, we see that Theorem 2.2 may be regarded as the "base case" for our inductive technique, with Theorem 2.3 and Theorem 2.4 providing the required evaluations for the latter two sums given above, thus highlighting the import and the utility of the theorems given in Section 2. Through an application of the technique described above, we obtain the following new results.

$$
\begin{aligned}
& \sum_{n=1}^{\infty} \frac{\left(\begin{array}{c}
2 n \\
n
\end{array}\right)^{2} H_{n}}{32^{n}(n+3)}= \\
& \frac{2048}{225}-\frac{4 \sqrt{\pi}(297 \pi+1188 \ln (2)-800)}{225 \Gamma\left(\frac{1}{4}\right)^{2}}- \\
& \frac{2 \Gamma\left(\frac{1}{4}\right)^{2}(313+25 \pi-100 \ln (2))}{225 \pi^{3 / 2}} \\
& \frac{\sum_{n=1}^{\infty} \frac{\left(\begin{array}{c}
2 n \\
n
\end{array}\right)^{2} H_{n}}{32^{n}(n+4)}=}{\frac{16384}{1225}+\frac{\Gamma\left(\frac{1}{4}\right)^{2}(-15974-1425 \pi+5700 \ln (2))}{3675 \pi^{3 / 2}}-} \\
& \frac{4 \sqrt{\pi}(-7984+2401 \pi+9604 \ln (2))}{1225 \Gamma\left(\frac{1}{4}\right)^{2}} \\
& \frac{\sum_{n=1}^{\infty} \frac{\left(\begin{array}{c}
2 n \\
n
\end{array}\right)^{2} H_{n}}{32^{n}(n+5)}=}{\frac{2097152}{99225}+\frac{2 \Gamma\left(\frac{1}{4}\right)^{2}(-1071611-98550 \pi+394200 \ln (2))}{297675 \pi^{3 / 2}}-} \\
& \frac{4 \sqrt{\pi}(-391872+102851 \pi+411404 \ln (2))}{33075 \Gamma\left(\frac{1}{4}\right)^{2}}
\end{aligned}
$$

We encounter computational obstacles in attempting to apply the same kind of inductive procedure with respect to summations of the form

$$
\sum_{n \in \mathbb{N}} \frac{\left(\begin{array}{c}
2 n \\
n
\end{array}\right)^{2} H_{n}}{32^{n}(2 n-2 z+1)}
$$


for $z \in \mathbb{Z}_{>0}$. This illustrates how the problem of computing generalizations of (1.7) can be very complicated and often requires a degree of ingenuity in the application of the fundamental lemma from [4]. The problem of evaluating

$$
\sum_{n=0}^{\infty} \frac{\left(\begin{array}{c}
2 n \\
n
\end{array}\right)^{2} H_{n}}{32^{n}(2 n-3)}
$$

is especially interesting, since there is a beautifully unexpected corollary of the symbolic evaluation for the infinite summation given above that we discuss in Section 5.

Suppose that we were to attempt to evaluate the series in (3.3) using Theorem 2.3, following the inductive strategy that had been employed in our generalization of the proof for Theorem 3.1. So, we must re-index (3.3) as demonstrated below.

$$
\begin{aligned}
& \sum_{n=1}^{\infty} \frac{32^{-n}\left(\begin{array}{c}
2 n \\
n
\end{array}\right)^{2} H_{n}}{2 n-3} \\
& \sum_{n=0}^{\infty} \frac{32^{-n-1}\left(\begin{array}{c}
2 n+2 \\
n+1
\end{array}\right)^{2} H_{n+1}}{2 n-1} \\
& \frac{1}{8} \sum_{n=0}^{\infty} \frac{2^{-5 n}(2 n+1)^{2}\left(\begin{array}{c}
2 n \\
n
\end{array}\right)^{2}\left(H_{n}+\frac{1}{n+1}\right)}{(n+1)^{2}(2 n-1)} \\
& \frac{1}{8} \sum_{n=0}^{\infty} \frac{2^{-5 n}(2 n+1)^{2}\left(\begin{array}{c}
2 n \\
n
\end{array}\right)^{2} H_{n}}{(n+1)^{2}(2 n-1)}+\frac{1}{8} \sum_{n=0}^{\infty} \frac{2^{-5 n}(2 n+1)^{2}\left(\begin{array}{c}
2 n \\
n
\end{array}\right)^{2}}{(n+1)^{3}(2 n-1)}
\end{aligned}
$$

However, modern computer algerba systems cannot evaluate the sum

$$
\sum_{n=0}^{\infty}\left(\frac{1}{32}\right)^{n} \frac{\left(\begin{array}{c}
2 n \\
n
\end{array}\right)^{2}(2 n+1)^{2}}{(n+1)^{3}(2 n-1)}
$$

in closed form. Moreover, if we apply partial fraction decomposition with respect to the factor

$$
\frac{(2 n+1)^{2}}{(n+1)^{2}(2 n-1)}
$$

in the "re-indexed" harmonic summation given above, we obtain the 
expression

$$
\frac{16}{9(2 n-1)}+\frac{10}{9(n+1)}-\frac{1}{3(n+1)^{2}},
$$

but it is not at all clear as to how the series

$$
\sum_{n \in \mathbb{N}} \frac{\left(\begin{array}{c}
2 n \\
n
\end{array}\right)^{2} H_{n}}{32^{n}(n+1)^{2}}
$$

could be evaluated, even through an application of the main lemma from [4], since this would require the symbolic computation of a difficult integral such as

$$
\int_{0}^{1} \frac{\ln (u) \ln \left(1+\frac{\sqrt{1+u}}{\sqrt{2}}\right)}{(1-u)^{3 / 2} \sqrt{u}} d u
$$

and it is not clear as to how to apply the Maclaurin series substitution strategy employed to evaluate $(2.2)$.

To evaluate series of the form

$$
\sum_{n \in \mathbb{N}} \frac{\left(\begin{array}{c}
2 n \\
n
\end{array}\right)^{2} H_{n}}{32^{n}(2 n-2 z+1)}
$$

for $z \in \mathbb{Z}_{>0}$, we may apply the following procedure inspired by our proofs for Theorem 2.2 and Theorem 2.3. We leave it as an exercise to verify the efficacy of this algorithm.

(1) Let $f(n)=\frac{2 n-1}{2^{n}(2 n-9)}$, apply the funamental lemma from [4], and evaluate the corresponding hypergeometric series

$$
\sum_{n \in \mathbb{N}} \frac{\left(\begin{array}{c}
2 n \\
n
\end{array}\right)^{2}}{32^{n}(2 n-2 z+1)}
$$

in closed form;

(2) Through the application of Lemma 1.1 noted above, we obtain an integrand of the form

$$
\sqrt{\frac{2-x^{2}}{1-x^{2}}} \ln \left(1-x^{2}\right) p(x)
$$

for a polynomial $p(x)$ with algebraic coefficients. Apply the substitution $u=1-x^{2}$; 
(3) We thus obtain an integrand of the form

$$
\sqrt{\frac{1+u}{1-u}} \cdot \frac{\ln (u)}{\sqrt{u}} \cdot q(u)
$$

for a polynomial $q(u)$ with algebraic coefficients. Replace the expression $\sqrt{\frac{1+u}{1-u}}$ with its Maclaurin series

$$
1+\sum_{n=1}^{\infty} 2^{1-n} u^{n}\left(\begin{array}{c}
n-1 \\
\left\lfloor\frac{n-1}{2}\right\rfloor
\end{array}\right)
$$

in the above integrand and integrate term-by-term.

Using the above procedure, we obtain the following results, thus illustrating the versatility of the main lemma from [4].

$$
\begin{aligned}
& \sum_{n=0}^{\infty} \frac{\left(\begin{array}{c}
2 n \\
n
\end{array}\right)^{2} H_{n}}{32^{n}(2 n-3)}= \\
& \frac{36 \Gamma\left(\frac{3}{4}\right)^{2}(\pi+4 \ln (2)-4)-\Gamma\left(\frac{1}{4}\right)^{2}(15 \pi-60 \ln (2)+8)}{216 \pi^{3 / 2}} \\
& \sum_{n=0}^{\infty} \frac{\left(\begin{array}{c}
2 n \\
n
\end{array}\right)^{2} H_{n}}{32^{n}(2 n-5)}= \\
& \frac{11 \pi^{3 / 2}}{75 \Gamma\left(\frac{1}{4}\right)^{2}-\frac{\Gamma\left(\frac{1}{4}\right)^{2}(32+51 \pi-204 \ln (2))}{1080 \pi^{3 / 2}}+\frac{4 \sqrt{\pi}(55 \ln (2)-51)}{375 \Gamma\left(\frac{1}{4}\right)^{2}}} \\
& \sum_{n=0}^{\infty} \frac{\left(\begin{array}{c}
2 n \\
n
\end{array}\right)^{2} H_{n}}{32^{n}(2 n-7)} \\
& \frac{\sqrt{\pi}(65 \pi+260 \ln (2)-212)}{875 \Gamma\left(\frac{1}{4}\right)^{2}}+\frac{\Gamma\left(\frac{1}{4}\right)^{2}(5796 \ln (2)-856-1449 \pi)}{41160 \pi^{3 / 2}}
\end{aligned}
$$

Using a similar algorithm, we may evaluate series of the form

$$
\sum_{n \in \mathbb{N}} \frac{\left(\begin{array}{c}
2 n \\
n
\end{array}\right)^{2} H_{n}}{32^{n}(2 n-2 z+1)^{2}}
$$


in closed form for $z \in \mathbb{Z}_{>0}$, and we leave it as an exercise to formalize this idea. For example, using an analogue of the procedure described above, we obtain the following result.

$$
\begin{aligned}
& \sum_{n=0}^{\infty} \frac{32^{-n}\left(\begin{array}{c}
2 n \\
n
\end{array}\right)^{2} H_{n}}{(2 n-3)^{2}}= \\
& \frac{4 \sqrt{\pi}(7-\pi-4 \ln (2))}{9 \Gamma\left(\frac{1}{4}\right)^{2}}+\frac{\Gamma\left(\frac{1}{4}\right)^{2}(8+7 \pi-28 \ln (2))}{216 \pi^{3 / 2}}
\end{aligned}
$$

4. Ramanujan-type formulas. Inspired by the method we had applied to generalize Theorem 3.1, we consider the use of similar strategies to determine explicit evaluations for new $\frac{1}{\pi}$ series with summands containing factors of the form

$$
\frac{\left(\begin{array}{c}
2 n \\
n
\end{array}\right)^{2} H_{n}}{16^{n}},
$$

which were the main kinds of mathematical objects under investigation in [4], as opposed to expressions of the form

$$
\frac{\left(\begin{array}{c}
2 n \\
n
\end{array}\right)^{2} H_{n}}{32^{n}}
$$

which served as something of a basis for Section 2 and Section 3. To illustrate this idea, we begin by considering the problem of finding a symbolic evaluation for the simple and natural-looking series

$$
\sum_{n=1}^{\infty} \frac{C_{n}^{2} H_{n}}{16^{n}},
$$

letting $C_{n}$ denote the $n^{\text {th }}$ Catalan number, as above. Summations of the form

$$
\sum_{n=1}^{\infty} \frac{\left(\begin{array}{c}
2 n \\
n
\end{array}\right)^{2} H_{n}}{16^{n}(n+z)^{2}}
$$

for $z \in \mathbb{Z}_{>0}$ had not been discussed in [4]; series of the form in (4.1) cannot be evaluated through a direct or straightforward application of Lemma 1.1, as is easily verified. We thus make use of a more "unusual" approach to prove the following result, which serves as the base case for an inductive generalization. 
Theorem 4.1. The following equation holds:

$$
\sum_{n=0}^{\infty} \frac{\left(\begin{array}{c}
2 n \\
n
\end{array}\right)^{2} H_{n}}{16^{n}(n+1)^{2}}=16+\frac{32 G-64 \ln (2)}{\pi}-16 \ln (2)
$$

Proof. We begin by making use of the following result that had been introduced in [4]:

$$
\sum_{n=1}^{\infty} \frac{\left(\begin{array}{c}
2 n \\
n
\end{array}\right)^{2} H_{n}}{16^{n}(2 n-3)}=\frac{-68+120 \ln (2)}{27 \pi}
$$

We remark that the above result follows directly from the fundamental lemma from [4] by letting $f(n)=\frac{2 n-1}{2 n-3}$. Now apply the following reindexing argument.

$$
\begin{aligned}
& \frac{-68+120 \ln (2)}{27 \pi}= \\
& \sum_{n=1}^{\infty} \frac{16^{-n}\left(\begin{array}{c}
2 n \\
n
\end{array}\right)^{2} H_{n}}{2 n-3}= \\
& \sum_{n=0}^{\infty} \frac{16^{-(n+1)}\left(\begin{array}{c}
2 n+2 \\
n+1
\end{array}\right)^{2} H_{n+1}}{2 n-1}= \\
& \frac{1}{4} \sum_{n=0}^{\infty} \frac{4^{-2 n}(2 n+1)^{2}\left(\begin{array}{c}
2 n \\
n
\end{array}\right)^{2} H_{n+1}}{(n+1)^{2}(2 n-1)}= \\
& \frac{1}{4} \sum_{n=0}^{\infty} 4^{-2 n}\left(\begin{array}{c}
2 n \\
n
\end{array}\right)^{2} H_{n}\left(-\frac{1}{3(n+1)^{2}}+\frac{10}{9(n+1)}+\frac{16}{9(2 n-1)}\right)+ \\
& \frac{1}{4} \sum_{n=0}^{\infty} 4^{-2 n}\left(\begin{array}{c}
2 n \\
n
\end{array}\right)^{2}\left(-\frac{1}{3(n+1)^{3}}+\frac{10}{9(n+1)^{2}}-\right. \\
& \left.\frac{16}{27(n+1)}+\frac{32}{27(2 n-1)}\right)= \\
& -\frac{1}{12} \sum_{n=0}^{\infty} \frac{\left(\begin{array}{c}
2 n \\
n
\end{array}\right)^{2} H_{n}}{16^{n}(n+1)^{2}}-\frac{1}{12} \sum_{n=0}^{\infty} \frac{\left(\begin{array}{c}
2 n \\
n
\end{array}\right)^{2}}{16^{n}(n+1)^{3}}-\frac{24 \ln (2)-40}{27 \pi}
\end{aligned}
$$

We remark that for the final equality given above, we implicitly made use of Lemma 1.1 in the case whereby $f(n)=1$. So, we have shown 
that the problem of symbolically computing the infinite series given in the above theorem is equivalent to the remarkably simpler problem of computing the hypergeometric series given below.

$$
\sum_{n=0}^{\infty} \frac{\left(\begin{array}{c}
2 n \\
n
\end{array}\right)^{2}}{16^{n}(n+1)^{3}}
$$

Surprisingly, Mathematica is not able to compute the above series. Making use of the Catalan number integral formula whereby

$$
\frac{\left(\begin{array}{c}
2 n \\
n
\end{array}\right)}{n+1}=\frac{1}{2 \pi} \int_{0}^{4} x^{n} \sqrt{\frac{4-x}{x}} d x,
$$

we find that the series in (4.2) is also equal to

$$
\begin{aligned}
& \frac{1}{2 \pi} \int_{0}^{4}-8 \sqrt{4-x}\left(\frac{1}{x}\right)^{3 / 2}(-2+\sqrt{4-x}+ \\
& \left.2 \ln (2)-2 \ln \left(1+\sqrt{1-\frac{x}{4}}\right)\right) d x
\end{aligned}
$$

which Mathematica is able to evaluate as $-\frac{32 G}{\pi}-16+\frac{48}{\pi}+16 \ln (2)$, thus completing our proof.

By analogy with our generalization of Theorem 3.1, we make use of the following procedure to compute infinite series of the form

$$
\sum_{n=0}^{\infty} \frac{\left(\begin{array}{c}
2 n \\
n
\end{array}\right)^{2} H_{n}}{16^{n}(n+z)^{2}}
$$

for $z \in \mathbb{Z}_{>0}$. Begin by rewriting (4.1) as follows.

$$
\begin{aligned}
& \sum_{n=1}^{\infty} \frac{\left(\begin{array}{c}
2 n \\
n
\end{array}\right)^{2} H_{n}}{16^{n}(n+z)^{2}}= \\
& \sum_{n=2}^{\infty} \frac{\left(\begin{array}{c}
2 n-2 \\
n-1
\end{array}\right)^{2} H_{n-1}}{16^{n-1}(n+z-1)^{2}}= \\
& 4 \sum_{n=2}^{\infty} \frac{4^{-2 n} n^{2}\left(\begin{array}{c}
2 n \\
n
\end{array}\right)^{2} H_{n}}{(2 n-1)^{2}(n+z-1)^{2}}-4 \sum_{n=2}^{\infty} \frac{4^{-2 n} n\left(\begin{array}{c}
2 n \\
n
\end{array}\right)^{2}}{(2 n-1)^{2}(n+z-1)^{2}}
\end{aligned}
$$


It is easily seen that

$$
\sum_{n} \frac{4^{-2 n} n\left(\begin{array}{c}
2 n \\
n
\end{array}\right)^{2}}{(2 n-1)^{2}(n+z-1)^{2}}
$$

has a closed-form expression for $z \in \mathbb{Z}_{>0}$, and we leave it as an exercise to show this. Through the use of partial fraction decomposition, expand the quotient

$$
\frac{n^{2}}{(2 n-1)^{2}(n+z-1)^{2}}
$$

as suggested below.

$$
\begin{aligned}
& -\frac{2 z-2}{(2 z-1)^{3}} \cdot \frac{1}{n+z-1} \\
& +\frac{1}{(2 z-1)^{2}} \cdot \frac{1}{(2 n-1)^{2}} \\
& +\frac{(z-1)^{2}}{(2 z-1)^{2}} \cdot \frac{1}{(n+z-1)^{2}} \\
& +\frac{4 z-4}{(2 z-1)^{3}} \cdot \frac{1}{2 n-1}
\end{aligned}
$$

Starting with Theorem 4.1 as the base case, we may thus evaluate (4.1) recursively since it is easily seen that series of the form

$$
\sum_{n=1}^{\infty} \frac{\left(\begin{array}{c}
2 n \\
n
\end{array}\right)^{2} H_{n}}{16^{n}(n+m)}
$$

for $m \in \mathbb{N}$ may be evaluated directly through Lemma 1.1. Following this algorithm, we obtain the new results indicated below.

$$
\begin{aligned}
& \sum_{n=1}^{\infty} \frac{\left(\begin{array}{c}
2 n \\
n
\end{array}\right)^{2} H_{n}}{16^{n}(n+2)^{2}} \\
& =\frac{112}{27}-\frac{64 \ln (2)}{9}+\frac{16(13+24 G-44 \ln (2))}{27 \pi} \\
& \sum_{n=1}^{\infty} \frac{\left(\begin{array}{c}
2 n \\
n
\end{array}\right)^{2} H_{n}}{16^{n}(n+3)^{2}}
\end{aligned}
$$




$$
\begin{aligned}
& =\frac{6272}{3375}-\frac{1024 \ln (2)}{225}+\frac{23632+30720 G-54208 \ln (2)}{3375 \pi} \\
& \sum_{n=1}^{\infty} \frac{\left(\begin{array}{c}
2 n \\
n
\end{array}\right)^{2} H_{n}}{16^{n}(n+4)^{2}} \\
& =\frac{129536}{128625}-\frac{4096 \ln (2)}{1225}+\frac{783408+860160 G-1484864 \ln (2)}{128625 \pi}
\end{aligned}
$$

5. Conclusion. Our explorations on the symbolic computation of series with summands containing

$$
\left(\frac{1}{32}\right)^{n}\left(\begin{array}{c}
2 n \\
n
\end{array}\right)^{2} H_{n}
$$

as a factor lead us to a very surprising discovery concerning a new series involving alternating harmonic numbers, as elaborated below. It seems that the problem of generalizing this discovery would form a promising area of research.

By Lemma 1.1, by letting $f(n)=\frac{2^{-n}(2 n-1)}{2 n-3}$, we find that the infinite series

$$
\sum_{n=0}^{\infty} \frac{32^{-n}\left(\begin{array}{c}
2 n \\
n
\end{array}\right)^{2} H_{n}}{2 n-3}
$$

is equal to the following.

$$
\begin{aligned}
& \frac{2}{\pi} \int_{0}^{1} \frac{\left(\sqrt{2-x^{2}}+x^{2} \sqrt{2-x^{2}}\right) \ln \left(1-x^{2}\right)}{3 \sqrt{2} \sqrt{1-x^{2}}} d x+ \\
& \frac{5 \Gamma\left(\frac{1}{4}\right) \ln (2)}{9 \sqrt{2 \pi} \Gamma\left(\frac{3}{4}\right)}+\frac{2 \sqrt{\frac{2}{\pi}} \Gamma\left(\frac{3}{4}\right) \ln (2)}{3 \Gamma\left(\frac{1}{4}\right)}
\end{aligned}
$$

Mathematica 11 is not able to evaluate the above expression in closed form. However, interestingly, if the function Simplify is applied to this expression, Mathematica produces the following output, after 11 minutes of computation, letting $\gamma$ denote the Euler-Mascheroni constant, and letting regularized hypergeometric functions be denoted 
with ${ }_{p} \tilde{F}_{q}$.

$$
\begin{aligned}
& \frac{1}{18 \sqrt{2} \pi}\left(\frac{10 \sqrt{\pi} \Gamma\left(\frac{1}{4}\right) \ln (2)}{\Gamma\left(\frac{3}{4}\right)}+\frac{24 \sqrt{\pi} \Gamma\left(\frac{3}{4}\right) \ln (2)}{\Gamma\left(\frac{1}{4}\right)}+\right. \\
& 3\left(4 \gamma \mathbf{E}(-1)+\pi\left(-\sqrt{\pi}\left(\left.2 \frac{\partial}{\partial x}{ }_{3} \tilde{F}_{2}\left[\begin{array}{c|c}
-\frac{1}{2}, \frac{1}{2}, \frac{1}{2} \\
1, x
\end{array} \mid-1\right]\right|_{x=\frac{1}{2}}+\right.\right.\right. \\
& \left.\frac{\partial}{\partial x}{ }_{3} \tilde{F}_{2}\left[\begin{array}{c}
-\frac{1}{2}, \frac{1}{2}, \frac{1}{2} \\
2, x
\end{array} \mid-1\right]\right|_{x=\frac{1}{2}}+ \\
& \left.2 \frac{\partial}{\partial x}{ }_{2} F_{1}\left[\begin{array}{c|c}
-\frac{1}{2}, \frac{1}{2} & -1 \\
x & -1
\end{array}\right]\right|_{x=1}+ \\
& \left.\left.\left.\frac{\partial}{\partial x}{ }_{2} \tilde{F}_{1}\left[\begin{array}{c|c}
-\frac{1}{2}, \frac{1}{2} & -1 \\
x &
\end{array}\right]\right|_{x=2}\right)\right)
\end{aligned}
$$

It can be shown that from the above output, together with the symbolic calculation for (5.1) that we had provided in Section 3, we have that the series

$$
\sum_{n=1}^{\infty}\left(-\frac{1}{16}\right)^{n}\left(\begin{array}{c}
2 n \\
n
\end{array}\right)^{2} \frac{(2 n+3) H_{2 n}^{\prime}}{(2 n-1)(n+1)}
$$

is may be evaluated as

$$
\frac{5 \Gamma\left(\frac{1}{4}\right)^{2}(\pi-4 \ln (2))}{12 \sqrt{2} \pi^{3 / 2}}-\frac{\Gamma\left(\frac{3}{4}\right)(3 \pi+12 \ln (2)-20)}{3 \sqrt{\pi} \Gamma\left(\frac{1}{4}\right)}-\frac{2}{3} .
$$

This is very interesting because it is unexpected that the main integration technique from [4] can also be applied to determine new series involving alternating harmonic numbers, and this suggests that there may be a deep connection between the fundamental lemma from [4] and the main integration technique from [5]. How can we obtain new classes of series containing harmonic-like numbers of the form $H_{2 n}^{\prime}$ as in (5.2) by showing how the definite integral in Lemma 1.1 can be expressed in terms of parameter derivatives of hypergeometric expressions of the form ${ }_{p} F_{q}(-1)$, as above?

\section{REFERENCES}

1. B.C. Berndt, Ramanujan's notebooks. Part I, Springer-Verlag, New York, 1985. 
2. J.M. Borwein and M. Chamberland, Integer powers of arcsin, Int. J. Math. Math. Sci. (2007), Art. ID 19381, 10.

3. K.N. Boyadzhiev, Series with Central Binomial Coefficients, Catalan Numbers, and Harmonic Numbers, J. Integer Seq. 15 (2012), Article 12.1.7.

4. J.M. Campbell, Ramanujan-like series for $\frac{1}{\pi}$ involving harmonic numbers, Ramanujan J. (2018) https://doi.org/10.1007/s11139-018-9995-9

5. J.M. Campbell and A. Sofo, An integral transform related to series involving alternating harmonic numbers, Integr. Transf. Spec. F. 28 (2017), 547-559.

6. H. Chen, Interesting series associated with central binomial coefficients, Catalan numbers and harmonic numbers, J. Integer Seq. 19 (2016), Article 16.1.5.

7. J. Choi, Summation formulas involving binomial coefficients, harmonic numbers, and generalized harmonic numbers, Abstr. Appl. Anal. (2014), Art. ID 501906, 10.

8. W. Chu and D. Zheng, Infinite series with harmonic numbers and central binomial coefficients, Int. J. Number Theory 5(3) (2009), 429-448.

9. M. Genčev, Binomial sums involving harmonic numbers, Math. Slovaca 61(2) (2011), 215-226.

10. J. Guillera, More hypergeometric identities related to Ramanujan-type series, Ramanujan J. 32(1) (2013), 5-22.

11. H. Liu, W. Zhou, and S. Ding, Generalized harmonic number summation formulae via hypergeometric series and digamma functions, J. Differ. Equations Appl. 23 (2017), 1204-1218.

12. S. Mattarei and R. Tauraso, Congruences for central binomial sums and finite polylogarithms, J. Number Theory 133(1) (2013), 131-157.

13. M. Nicholson, Quadratic Transformations of Hypergeometric Function and Series with Harmonic Numbers, arXiv: 1801.02428

14. Z.-W. Sun, p-adic congruences motivated by series, J. Number Theory 134 (2014), 181-196.

15. Z.-W. Sun, A new series for $\pi^{3}$ and related congruences, Internat. J. Math. 26(8) (2015), 1550055, 23.

16. Z.-W. Sun, New series for some special values of L-functions, Nanjing Univ J Math. 32(2) (2015), 189-218.

17. R. Tauraso, Supercongruences related to ${ }_{3} F_{2}(1)$ involving harmonic numbers, Int. J. Number Theory (2017), 1-17. arXiv:1701.00729

18. J.G.F. Wan, Random walks, elliptic integrals and related constants, $\mathrm{PhD}$ Thesis, University of Newcastle, Newcastle, 2013.

York University, Department of Mathematics and Statistics, Toronto, CANADA

Email address: jmaxwellcampbell@gmail.com 